\title{
Fungi in soil and understory have coupled distribution patterns
}

\author{
André Boraks ${ }^{\text {Corresp., } 1}$, Anthony S Amend ${ }^{1}$ \\ ${ }^{1}$ School of Life Science, University of Hawaii at Manoa, Honolulu, Hawai'i, United States \\ Corresponding Author: André Boraks \\ Email address: aboraks@hawaii.edu
}

Ecological processes that control fungal distribution are not well understood because many fungi can persist in a wide variety of dissimilar habitats which are seldom sampled simultaneously. Geographic range size is reflective of species' resource usage, and for plants and animals, there is a robust positive correlation between niche-breadth and range-size. It remains unknown whether this pattern is true for fungi. To investigate the fungal niche breadth-range size relationship we identified habitat specialists and generalists from two habitats (plant leaves and soil) and asked whether habitat specialization influenced fungal biogeography. We sampled fungi from the soil and phylloplane of tropical forests in Vanuatu and used DNA metabarcoding of the fungal ITS1 region to examine rarity, range size, and habitat connectivity. Fungal communities from soil and the phylloplane are spatially autocorrelated and the spatial distribution of individual fungal OTU are coupled between habitats. Habitat breadth (generalist fungi) did not result in larger range sizes but did correlate positively with occurrence frequency. Fungi that were frequently found were also found in high abundance, a common observation in similar studies of plants and animals. Fungal abundance-occupancy relationships differed by habitat and habitat-specificity. Soil specialists were found to be locally abundant but restricted geographically. In contrast, phylloplane generalists were found to be abundant over a large range in multiple habitats. These results are discussed in the context of differences between habitat characteristics, stability and spatial distribution. Identifying factors that drive spatial variation is key to understanding the mechanisms that maintain biodiversity in forests. 


\section{Fungi in soil and understory have coupled distribution patterns}

2

3

4

5

6

\section{Abstract}

Ecological processes that control fungal distribution are not well understood because many fungi can persist in a wide variety of dissimilar habitats which are seldom sampled simultaneously. Geographic range size is reflective of species' resource usage, and for plants and animals, there is a robust positive correlation between niche-breadth and range-size. It remains unknown whether this pattern is true for fungi. To investigate the fungal niche breadth-range size relationship we identified habitat specialists and generalists from two habitats (plant leaves and soil) and asked whether habitat specialization influenced fungal biogeography. We sampled fungi from the soil and phylloplane of tropical forests in Vanuatu and used DNA metabarcoding of the fungal ITS1 region to examine rarity, range size, and habitat connectivity. Fungal communities from soil and the phylloplane are spatially autocorrelated and the spatial distribution of individual fungal OTU are coupled between habitats. Habitat breadth (generalist fungi) did not result in larger range sizes but did correlate positively with occurrence frequency. Fungi that were frequently found were also found in high abundance, a common observation in similar studies of plants and animals. Fungal abundance-occupancy relationships differed by habitat and habitat-specificity. Soil specialists were found to be locally abundant but restricted geographically. In contrast, phylloplane generalists were found to be abundant over a large range in multiple habitats. These results are discussed in the context of differences between habitat characteristics, stability and spatial distribution. Identifying factors that drive spatial variation is key to understanding the mechanisms that maintain biodiversity in forests.

\section{Introduction}

The physical and temporal characteristics of a habitat influence the geographic distribution of its inhabitants (Brown, Stevens \& Kaufman, 1996; Clark et al., 2021). Forest ecosystems contain numerous habitats, many of which are found in close proximity and nearly all of which host rich fungal communities. Fungi are cosmopolitan and are found in the soil, rhizosphere, roots, plant litter, foliage, bark, wood, rocks, and water (Větrovský et al., 2020). The particular set of features unique to each habitat drives differences in fungal diversity among habitats (Baldrian, 2017) such that the composition of fungal communities vary predictably even though habitats typically contain a mixture of both habitat specialists and habitat generalists (Amend et al., 2019). Habitat breadth and range size is positively correlated for plants and animals (Slatyer, Hirst \& Sexton, 2013) but the correlation between fungal habitat breadth and rarity remains unknown.

Range size is the basic unit of biogeography. Differences in range size among species provide insight into processes of adaptation and dispersal (Pulliam, 2000). Range size and niche breadth represent two of the three major axes of commonness and rarity (Rabinowitz, 1981) and are strong predictors of extinction risk (Chichorro, Juslén \& Cardoso, 2019). Brown (1984) argued that species utilizing greater number of resources and persisting among a greater variety of environmental conditions would become more widespread. Brown's resource-use hypothesis remains a central topic in ecology (Slatyer, Hirst \& Sexton, 2013; Sheth, Morueta-Holme \& 
44 Angert, 2020) but it is seldom examined for microbes, especially fungi. A recent study of plant associated fungi showed that habitat breadth was a strong predictor of the range size over a $5 \mathrm{~km}$ gradient (Bernard et al., 2020). These preliminary results suggest that fungi adhere to similar niche breadth - range size correlations that are observed in plants and animals.

As a means for understanding mechanisms of biogeographic patterns, range size lacks the resolution of detailed distribution maps. No standard methodology exists for measuring the area over which a species is found and determining the area of occupancy implies an understanding of the species' habitat breadth (Gaston \& Fuller, 2009). For habitat specialists this may be somewhat straightforward because field sampling can focus on mapping species distributions within a targeted habitat. Fungi are problematic in this regard because species can be recovered from multiple distinct habitats. Within a forest, Aspergillus niger has been isolated from air, deadwood, litter, rhizosphere, root, soil, sediment, and plant shoots (Větrovský et al., 2020). Over $60 \%$ of the fungi isolated as endophytes can also be isolated from leaf litter (Osono, 2006), implying that a researcher would need to consider the linked distributions among all potential habitats to effectively measure a species' range. Linking species distribution maps from more than one habitat provides a novel understanding of how fungi are distributed within and between habitats.

62

The phylloplane and soil differ in their spatial and temporal dynamics. Leaves have a comparatively short lifespan and can be visualized as habitat islands. Soil, in contrast, is contiguous and can persist indefinitely unless disturbed. Living among both of these habitats are habitat generalists (fungal taxa that occur in multiple habitats) and a set of habitat specialists (fungi that occur exclusively in a single habitat). Soil is one of the most frequently studied forest habitats due to the ubiquity of belowground symbiotic relationships, like mycorrhizae, and the importance of belowground ecosystem processes including carbon and nutrient cycling (Baldrian, 2017). Aboveground, the phyllosphere occupies greater than 1 billion $\mathrm{km}^{2}$; an area more than double the earth's land surface (Vorholt, 2012). Microbes of the phylloplane modify plant performance and regulate plant diversity, factors that ultimately result in cascading effects on ecosystem functioning (reviewed in (Vorholt, 2012; Griffin \& Carson, 2018)). The two habitats have unique and distinct characteristics. Plant leaves have a waxy cuticle layer that reduces moisture and nutrient loss resulting in an oligotrophic surface. Plant leaves experience large fluctuations in diurnal temperature, moisture, wind abrasion, and UV radiation (Vorholt, 2012). By comparison, diurnal moisture and temperature regimes are more stable in the soil. Soil and the phylloplane are separate but adjacent habitats that each harbor rich fungal communities.

Fungi are generally sessile organisms that move about the environment by spore dispersal and hyphal growth. Large and contiguous habitats allow fungal mycelium to propagate for long timespans and great distances. Such is the case for soil dwelling Armalaria ostoyae which have grown to become some of the oldest and largest organisms on earth (Ferguson et al., 2003). It is 
84 well established that soil is neither uniform nor static, although given sufficient conditions, soil-

85 dwelling fungi can spread impressive distances via mycelial growth. Despite the potential to

86 spread indefinitely via mycelium, fungi primarily disperse about the environment by spores (both

87 meiotic and mitotic). Unlike mycelial growth, spore dispersal allows fungi to disperse and

88 establish on disconnected habitat patches (Halbwachs \& Bässler, 2015), like separate plant

89 leaves. Mycelial growth on plant leaves is restricted in distance by the physical dimensions of the

90 leaf, so it would be advantageous for foliar fungi to also be prolific spore dispersers. Life in the

91 phylloplane offers fungi exceptional access to the vectors that disperse spores, but because of the

92 discontinuity and ephemerality of the habitat, dispersal is uncertain. In comparison to the

93 phylloplane, hypogeous soil-dwelling fungi have access to a habitat that is larger more and

94 temporally stable and as a result have the potential to spread great distances via mycelial growth

95 or even remain dormant in the spore bank for many years (Bruns et al., 2009; Nguyen, Hynson \&

96 Bruns, 2012). Previous research comparing soil and airborne fungal communities found soil

97 communities were primarily correlated with abiotic parameters, compared to airborne

98 communities that shifted temporally (Kivlin et al., 2014). Differences in parameters affecting

99 communities in these two habitats brought us to question whether habitat type influenced fungal

100 range size.

101

102 We suspected that fungal communities from the phylloplane and soil to be spatially related

103 because phylloplane fungi are transported vertically by senescent leaves that fall to the soil

104 below. In this study we present a spatial perspective on the similarities of phylloplane and soil

105 mycobiomes. If there is community membership overlap among the soil and phylloplane, is the

106 overlap spatially autocorrelated? Range size has is often correlated with niche breadth (Slatyer,

107 Hirst \& Sexton, 2013; Bernard et al., 2020) so we investigated the relationship between habitat

108 specificity and range size. Range size can refer to a number of different measurements (Brown,

109 Stevens \& Kaufman, 1996), so for the purpose of this study range size is the geographic area

110 occupied by an OTU within a transect. Do the range sizes of habitat generalists and habitat

111 specialists differ for leaves and soil? Finally, does habitat type influence range size? Forest

112 dwelling fungi occupy a variety of different habitats, here we explore the relationship between

113 habitat occupancy, connectivity, and fungal biogeography.

114

\section{Materials \& Methods}

116 Study site

117 Field sampling consisted of two separate forays in August 2017 (Aneityum) and in December

1182017 (Tanna) in the province of Tafea, Vanuatu as previously described (Boraks et al., 2020).

119 The two islands are separated by 86 kilometers of open ocean and share a similar tropical

120 climate. Aneityum receives $2322 \mathrm{~mm}$ of rain annually and has an average annual temperature of

$12119.7^{\circ} \mathrm{C}$, although there is large seasonal and interannual variation (Vanuatu Meteorology and

122 Geo-hazard Department, Australian Bureau of Meteorology \& CSIRO, 2015). Our sampling 
123 locations are classified as low to mid-elevation rain forest (Mueller-Dombois \& Fosberg, 2013)

124 dominated by Syzygium, Dysoxylum, and Hedycarya species (Boraks et al., 2020).

125

126 Six forest transects (10 m by $40 \mathrm{~m}$ ) were sampled, three on each island (Fig. S1). Within each

127 transect we sampled 36 locations in grid formation. At each sampling location, we collected the

128 fungal community from both the soil and the leaf surface (phylloplane). Fungal communities

129 were harvested using flocked sterile swabs and preserved in CTAB buffer. Soil samples were

130 collected by brushing away loose plant litter and inserting swabs into the organic layer of topsoil.

131 Phylloplane samples were collected by swabbing leaf surfaces. Leaf sampling height varied by

132 availability, but generally were located between 1 and 3 meters above the forest floor. The total

133 area sampled for each phylloplane sample was constrained to a surface area of $400 \mathrm{~cm}^{2}$, or

134 roughly the surface area of two hands. Plant taxonomy influences the phyllopsphere mycobiome

135 to a greater (Kembel \& Mueller, 2014), or lesser (O'Rorke et al., 2015), degree and we attempted

136 to reduce this variance contribute by swabbing leaves from multiple plant species using the same

137 swab. We aimed to include at least three different plant species per sampling site.

138

139 Soil and phylloplane samples were taken in pairs from the same geographic location, such that

140 the phylloplane-sampling grid and the soil-sampling grid were coupled. This paired-habitat and

141 spatially explicit sampling scheme totaled 432 fungal community samples (36 sampling sites x 2

142 habitats x 6 transects) (Boraks et al., 2020).

143

\section{DNA extraction, PCR and sequencing}

145 Sequencing library preparation was previously described in (Boraks et al., 2020).

146 DNA was extracted from the swabs using the Qiagen dneasy powersoil dna isolation Kit

147 (Qiagen, Maryland). We slightly modified the extraction protocol by extracting directly from

148 swabs. Amplicon libraries were prepared in a single PCR reaction using Illumina-barcoded

149 fungal-specific primers as in Amend et al. (2019). The primers ITS1F and ITS2 were used to

150 target the hypervariable nuclear ribosomal ITS1 region which is flanked by the 18S and 5.8S

151 rDNA regions (Schoch et al., 2012). Sequence library was purified and normalized (Just-a-plate,

152 Charm Biotech), quantified by qPCR and sequenced on Illumina MiSeq platform using V3

153 chemistry (Illumina Inc., San Diego, CA) at the Institute for Integrative Genome Biology

154 (University of California-Riverside). Additional details of sequence library prep can be found in

155 Boraks et al. (2020). Sequences are deposited in the NCBI Sequence Read Archive (BioProject:

156 PRJNA634909)

157

158 ITS1 sequences were extracted from the flanking ribosomal subunit genes using ITSxpress

159 (Rivers et al., 2018), then filtered by quality scores using the FASTX-Toolkit (Gordon \& Hannon,

160 2010). Reverse sequence reads were discarded because of lower quality reads. Chimeras were

161 detected and removed using vsearch (Rognes et al., 2016). Sequences were clustered at 97\%

162 identity and fungal taxonomy was assigned using the Python package constax (Gdanetz et al., 
163 2017) and the unite 8.0 database. Putative contaminants were identified based on their

164 prevalence in extraction and PCR negative controls and removed using the R package decontam

165 (Davis et al., 2017). OTUs that could not be assigned to a fungal phylum were discarded. The

166 sequence library was normalized by variance stabilizing transformation (VST) (McMurdie \&

167 Holmes, 2014) in DESeq2 (Love, Huber \& Anders, 2014) within R (R Core Team, 2019).

168 Singletons were removed for all analyses except those that measured community dissimilarity.

169 Sequence read abundance data, taxonomic assignments, sample metadata, and ancillary

170 collection data were compiled with the R package phyloseq (McMurdie \& Holmes, 2013). Using

171 sequence read abundance as a metric for species abundance is contentious due to the

172 compositional nature of high-throughput sequencing datasets (Gloor et al., 2017). Statistical

173 analyses for this study rely on both presence-absence and sequence abundance data. Caution

174 should be exercised when interpreting analyses that use relative sequence read abundance as

175 metric for OTU abundance.

176

177

178

Statistical analyses

Are fungal communities different between habitats?

179 Phylloplane and soil fungal communities were compared using NMDS ordination (using Bray-

180 Curtis dissimilarity) and permutational analysis of variance (permanova) as implemented in the

181 Vegan package (Oksanen et al., 2013). The permanova (adonis) was iterated with 999

182 permutations.

183

184

\section{Inter-habitat spatial autocorrelation}

185 We tested for spatial autocorrelation in OTU co-occurrence between habitats. Bray-Curtis

186 community dissimilarity measurements assessed whether samples collected from different

187 habitats, but from the same geographic location, were more similar than pairwise draws of

188 disjunct locations and habitats. Our null hypothesis was that differences in community

189 composition were independent of habitat and geographic distance. This analysis was conducted

190 in a pairwise manner and a student t-test was calculated. The analysis was then performed at two

191 different spatial scales. The two spatial scales tested differed in grain size: the first tested for

192 inter-habitat spatial autocorrelation by comparing point-samples across the extent of our whole

193 study (both islands $\sim 110 \mathrm{~km}$ ), whereas the second test measured spatial autocorrelation between

194 point-samples and the extent of a transect $(40 \mathrm{~m})$.

195

196 Area of occupancy

197 To compare range sizes between habitats (soil or phylloplane) and lifestyle (specialist or

198 generalist), we first categorized OTUs by habitat and lifestyle. Habitat specialization was

199 assigned if an OTU was found more than once and exclusively in a single habitat type, whereas

200 habitat generalists occurred more than once in each habitat from the same transect (Fig. S2).

201 Occupancy was calculated by the number of samples in which an OTU occurred within a

202 transect. This analysis was constrained within transects so the maximum occupancy was limited 
203 to the number of sampling points within a transect (36 locations, or roughly $400 \mathrm{~m}^{2}$ ). A

204 Wilcoxon rank sum test, corrected for multiple hypothesis testing (Benjamini \& Hochberg,

205 1995), was used to compare median range size.

206

207

Range size per substrate and specialization

208 To measure the range size of individual OTUs, we identified those that were spatially

209 autocorrelated using a Mantel test with Bray-Curtis distances based on OTU relative sequence-

210 read abundance and then built empirical semi-variogram models for each spatially autocorrelated

211 OTU. Semi-variogram models are useful for analyzing the range of variation between geospatial

212 phenomena. In this study we used semi-variograms to measure the geospatial decay of OTU

213 relative sequence read abundance. Semi-variogram models were developed for each spatially

214 autocorrelated OTU and geographic-range sizes were estimated from the range of each

215 variogram model. The nugget (zero) and model type (exponential) were fixed for all variograms

216 to increase comparability of range size between models. Range sizes were aggregated by habitat

217 and specialization and then compared by pairwise Wilcoxon tests corrected for multiple

218 hypothesis testing. Kriged surface maps were paired for generalist OTU that occurred in both

219 habitats of the same transect.

220

221

222

Occupancy-abundance relationships

223

To compare OTU rarity, we generated occupancy-abundance (OA) regressions for all OTUs of

224

each habitat and then compared linear regressions (loess fit) for specialists and generalists of the soil and phylloplane. OTUs were first categorized by habitat and specialization, and then the

225 number of occurrences per transect was tallied for each OTU. This type of OA regressions is

226

227 known as interspecific occupancy-abundance relationships because the regressions compare multiple OTUs. The maximum occupancy of any single OTU is 36 , because there were 36

228 sampling locations per transect. Abundance was measured using normalized sequence reads.

229

230

\section{Results}

231

232

Are fungal communities different between habitats?

233

When all transects were combined, 4949 OTUs were identified in the soil and 5362 in the

234 phylloplane. Ten OTUs were previously identified as suspect contaminats and were removed from the dataset. Transects contained an average of 4415 OTUs (standard error of mean (SE)

$235 \pm 340 ; \mathrm{n}=6)$. On average, $55 \%(\mathrm{SE} \pm 7 \%)$ of the OTUs that were present in a transect could be

236 found in the soil habitat. This was similar for phylloplane, in which $65 \%(\mathrm{SE} \pm 5 \%)$ of the OTUs

237 that were present in a transect could be found in the phylloplane. Permanova indicated

238 significant differences $(p<0.001)$ in community composition between soil and phylloplane

239 (habitat $\left.\mathrm{R}^{2}=0.11, \mathrm{p}<0.001\right)$ and island $\left(\mathrm{R}^{2}=0.03, \mathrm{p}<0.001\right.$; Fig. 1a).

240

241

Inter-habitat spatial autocorrelation 
242 Composition of fungal communities of the soil and the phylloplane differ, yet $22 \%$ of OTUs co243 occur in both habitats (generalists). Co-occurrence between habitats was spatially autocorrelated at a large scale $(110 \mathrm{~km})$, phylloplane communities were more similar to proximate soil communities than distant soil communities (Table 1). At a smaller scale $(<40 \mathrm{~m})$ the spatial autocorrelation of fungal communities from different habitats is less supported. Inter-habitat spatial autocorrelation was statistically significant for three of the six transects (Table 1). An OTU was categorized as a habitat specialist if it was found more than once and exclusively among a single habitat type. We identified 2187 soil specialist OTUs and 2212 phylloplane specialist OTUs (Fig. 1b). We defined OTU generalists as those occurring more than once in each habitat. A total of 1307 unique OTUs were classified as generalists. Thirty-five percent of the generalist OTUs occurred in more than one transect. Generalist fungi had higher occupancy rates than specialist fungi (Fig. 2). Median occupancy rates were greater for generalist fungi (phylloplane generalist $\mathrm{mdn}=6$, iqr $=12$; soil generalist $\mathrm{mdn}=4$, iqr $=6$ ) than for specialist fungi (phylloplane specialist $\mathrm{mdn}=3$, iqr $=3$; soil specialist $\mathrm{mdn}=3$, iqr $=4$ ) $($ Fig. 2).

258

259

\section{Semi-variogram modeling}

260

261

Spatial autocorrelation was detected among $7.7 \%$ of the phylloplane specialists and $10.4 \%$ of the soil specialists. Spatial autocorrelation was detected among $10.5 \%$ of the phylloplane generalists and $18.8 \%$ of soil generalists. Range sizes were modeled using experimental variograms that were based on the number of sequence reads as a function of spatial distance. A total of 1060 variogram models were generated. Variogram models were manually inspected, and we culled models $(515 / 1060)$ that reported unrealistically small ranges $(<1$ meter) for our given sampling scheme. In addition, 6 OTUs were identified as outliers and, despite robust variogram models, had exceptionally large ranges and were removed from downstream analysis (Table S1). The six outliers with large range sizes (mean $=737$ meter) included five OTUs within the Ascomycota and one OTU within Glomeromycotina. The removal of unreliable models and outliers resulted in 539 variograms remaining. Range size of specialist soil fungi (2.78 meter; iqr 1.77) were significantly greater than phylloplane generalist fungi (2.11 meter; iqr 1.53) and phylloplane specialist fungi $(2.02$ meter; iqr 1.82) $(\mathrm{p}<0.05)$. Soil generalist fungi $(2.54$ meter; iqr 1.66$)$ did not significantly differ in range size from soil specialists or phylloplane generalists (Fig. 3). Including the culled outliers OTU did not affect the significance of range-size comparisons. distances greater than 5 meters, although several ranges were estimated to be hundreds or thousands of meters.

278

\section{Distribution maps for generalist OTU}

280 Kriged surface maps were produced for 11 OTUs that were identified as spatially-autocorrelated generalist fungi which occurred in the same transect. The OTUs were identified as 
282 OTU97_1160_Dothideomycetes sp.; OTU97_1992_Helotiales sp,;

283 OTU97_2240_Agaricomycetes sp.; OTU97_228_Psathyrella sp.; OTU97_257_Polyporale sp.;

284 OTU97_2608_Xylariales sp.; OTU97_2833_Agaricales sp.; OTU97_3832_Psathyrellaceae sp.;

285 OTU97_3832_Psathyrellaceae sp.; OTU97_5320_Xylariales sp.; OTU97_6890_Ascomycota sp.

286 In general, the spatial distribution of an OTU was synchronous between habitats (Fig. 4). Semi-

287 variogram models and kriging standard errors are reported in the supplemental (Fig. S3)

288

289

\section{Occupancy-abundance relationships}

290

291

Phylloplane and soil occupancy-abundance relationships followed classical biogeographical expectations; the number of samples in which an OTU occurred was positively correlated with

292 the mean abundance of that OTU (Fig. 5). Among the phylloplane, specialists and generalists had similar abundance-occupancy regression slopes at low occupancy levels, but the regressions

294 diverged at high occupancy levels (Fig. 5). Common phylloplane generalists were more abundant than common phylloplane specialists. Among the soil, low-occupancy specialist fungi were more abundant than low occupancy generalists, but specialist and generalist abundance converge at higher occupancy rates (Fig. 5).

298

299

\section{Discussion}

300

Co-occurrence among habitat types

301

Here, we report a first attempt to couple distribution maps of fungal OTUs in the soil and

302 understory. These map stacks provide a rich visualization of where generalist fungi persist simultaneously in either habitats across the forest landscape (Fig. 4). In addition to the visual evidence, inter-habitat spatial autocorrelation confirmed that fungal communities of the phylloplane are more likely to be similar to nearby soil communities than to distant soil communities (Table 1). The inter-habitat spatial autocorrelation observed here was especially pronounced at large spatial scales $(<110 \mathrm{~km})$ but became less evident at smaller spatial scales $(<$ $40 \mathrm{~m}$ ). Stated differently, this result indicates the possibility of predicting the geo-location of a leaf surface based on the fungal community of soil samples (or vice versa), and that the accuracy of this prediction becomes less reliable at small spatial scales $(<40 \mathrm{~m})$.

311

312 Migration between habitats is important for community turnover of ephemeral habitats such as pools of water, phytotelma, and plant leaves. Leaf age is directly correlated with mycobiome

314 composition. Young, newly emergent plant leaves are sparsely colonized by fungi (Wildman \&

315 Parkinson, 1979; Suryanarayanan \& Thennarasan, 2004; Oono et al., 2015). The initial

316 colonization is a stochastic processes via horizontally transmitted fungi from unknown

317 environmental sources (Unterseher et al., 2018). As the leaf ages, fungal infection increases and

318 beta-diversity decreases (Oono et al., 2015). The decrease in beta-diversity may be partially

319 driven by host identity (Unterseher et al., 2013; Kembel \& Mueller, 2014; O'Rorke et al., 2015).

320 We attempted to compensate for the effect of host identity by sampling the leaves of multiple

321 plant hosts from the same sampling location. After a leaf has senesced and fallen from the plant, 
322 it will carry a rich microbial community to the forest floor. The phylloplane and soil are

323 physically disjunct habitats but spatially autocorrelated overlap in their mycobiomes (Table 1) is

324 suggestive of migration between them.

325

326

It is well established that microbes from the forest floor migrate to the phylloplane and vice

327 versa. Indeed, this is the main tenant of the latent saprotroph hypothesis which proposes that a

328 saprotroph is able to colonize the living tissue of its host to gain competitive advantage when the host tissue begins to senesce (Porras-Alfaro \& Bayman, 2011; Peršoh, 2013). Phytopathology literature contains examples of foliar pathogens that overwinter in ground litter, only to migrate back to the phylloplane when conditions are optimal. For example, the tree pathogen Venturia inaequlis is a hemibiotroph and must spend part of its lifecycle as a parasite, and part of its lifecycle on the dead tissue of the same host as a saprotroph (Agrios, 2004). In line with our results $(59 \%, \mathrm{n}=3519)$, a previous report examining the overlap of phyllosphere and leaf litter found $64 \%$ of leaf epiphytes could also be isolated from leaf litter (Osono, 2006). An important distinction in our study is that we sampled from the organic soil layer rather than leaf litter. Evidence from bacterial (Bai et al., 2015; Zarraonaindia et al., 2015; Wagner et al., 2016) and fungal systems (Amend et al., 2019; Bernard et al., 2020) have shown that soil may act as a reservoir of microbes that migrate to the phylloplane, an observation that is supported by our results (Table 1).

\section{Habitat breadth and range size}

Range size and niche breadth are positively correlated among plants and animals (Slatyer, Hirst \& Sexton, 2013; Bernard et al., 2020). Here we show that for fungi, niche breadth may or may not correlate with range size, and that this equivocal result depends on how range size is calculated (Fig. 2 and 3). Range size and habitat specificity are two of the major axes of rarity (Rabinowitz, 1981) and by including sequence read abundance we explore commonness and rarity of fungi from the phylloplane and soil.

There is no standard methodology for measuring species' geographic range. The large suite of techniques available can capture fundamentally distinct features of species distribution (Gaston, 1996). We used two different approaches to measure fungal geographic range size. First, we considered area of occupancy per transect (Fig. 2). This is a tally of the number of sampling-sites at which an OTU is recorded within a transect. Calculating range size by areas occupied is advantageous because of its simplicity, it requires only presence-absence data and it is inclusive of rare species (Gaston, 2008), and was successfully used in a study of bacterial range size (Choudoir et al., 2018). The drawback to this approach is that it lacks distribution nuance; it is unable to differentiate between a sparse and clustered distribution of the same occupancy level. The second method we used to measure range size was variography which provided a measurement most similar to Gaston's (1996) geometric circle (Fig. 3). In this study the radius a range was calculated from the range of an empirical semi-variogram. This technique was 
362 previously used in a study of ectomycorrhizal fungi (Pickles et al., 2010). Another way to think

363 of this measurement is to consider the linear distance, from the geometric center of a range, at

364 which variation in OTU sequence abundance is no longer spatially autocorrelated. The advantage

365 of the variogram approach is that it provides a linear distance measurement which can be used to

366 make visually illustrative maps (Fig. 4). When interpreting kriged variogram maps (Fig 4)

367 generated using high throughput sequencing data, it is important to consider their limitations.

368 Variogram modeling is only possible on a small fraction of our metabarcoding dataset (Fig. S2)

369 because of the large amounts of spatially autocorrelated data required and most OTU datasets are

370 dominated by low-abundance taxa. Relative sequence read abundance is compositional and

371 therefore comparisons between samples may be misleading (Gloor et al., 2017; Morton et al.,

372 2019). Additionally, amplicon sequencing is indiscriminate and we are unsure whether we

373 sequenced spores, hyphae, or exogenous DNA (Carini et al., 2016).

374

375

Brown (1984) affirmed that species which have a broad environmental range and are able to use

376 a wide range of resources will be both locally abundant and widespread. For fungi, adjacency of

377 steppingstones within a habitat matter little if you can just as easily step between habitats. In an

378

379 analysis of soil bacteria, Luo et al. (2019) found that generalist bacteria occurred at greater abundances than specialist bacteria. Results from our area of occupancy analysis support theoretical expectations insofar as we found that generalist fungi occur more frequently than specialists (Fig. 2). Interestingly, greater transect occupancy levels did not translate into larger modeled range sizes. Variogram estimations indicated no significant differences in range sizes between habitat specialists and generalists (Fig. 3). Lack of difference in the variogram range sizes might be attributable to the insensitivity of variograms to low occupancy OTU. Differences in range size results from each technique highlights the complicated nature of spatial distribution and habitat specialization.

A positive interspecific abundance-occupancy relationship is one of the most widely observed patterns in macroecology. We tested for variation in abundance-occupancy regression slopes between specialists and generalists from two separate habitats. OTUs of this study exhibited a positive occupancy-abundance relationship which describes the greater-than-proportional increase in abundance with respect an increase in occupancy (Fig. 5). When transect occupancy is low, soil specialists are more abundant than soil generalists (Fig. 5). We believe this may be result of the comparative stability of soil. Without significant perturbation, soil microbial communities show limited turnover (Kivlin et al., 2014; Žifčáková et al., 2016) potentially due to the longevity of spore banks (Bruns et al., 2009; Nguyen, Hynson \& Bruns, 2012). In less disturbed habitats, a strong competitor can persist in one location, cumulatively adding to the volume of spores within the spore bank of that location. This process might explain the pattern of locally abundant soil specialists towards the left side of Fig. 5b. The result of this study are similar to a previous report that belowground fungi had high local abundance but small species ranges, while aboveground fungi had lower local abundance but larger spatial ranges (Kivlin et 
402 al., 2014). Generalists occur more commonly and abundantly among the phylloplane, whereas in

403 the soil specialist fungi were found at high abundance but low occupancy (Fig. 5). By contrasting

404 the physical and temporal characteristics of these two habitats we were able to infer potential

405 strategies employed by fungi to persist on the landscape.

406

407

\section{Conclusions}

408 Geographic range size is a basic unit of biogeography, and its positive correlation with niche-

409 breadth is a well-established phenomenon observed among plants and animals. The importance

410 of this correlation cannot be understated; it is proposed as a mechanism for rarity, has

411 implications for extinction risk, and may provide insight into processes of adaptation and

412 dispersal. We demonstrate inter-habitat spatial autocorrelation of fungal communities, an

413 indication of fungal dispersal between soil and the phylloplane. Additionally, fungi of differing

414 habitats have similar biogeographic patterns. Local range size for fungal OTUs rarely exceed 5

415 meters. Habitat generalists and specialists have different occupancy-abundance models, an

416 indication that the effects of specialization on range size is habitat dependent. The phylloplane

417 and soil are habitats with dissimilar temporal and environmental characteristics and this study

418 provides important insights of fungal distribution within and between these two habitats.

419 Phylloplane generalist fungi have larger range sizes than phylloplane specialist fungi. This not

420 true for soil dwelling fungi. Soil specialization is associated with smaller range sizes and greater

421 abundances. Fungal biogeography is determined by the unique spatial and temporal

422 characteristics of the habitat.

423

424 Acknowledgements

425 This project would not be possible without the hard work and dedication from the ever-growing

426 network of people associated with Plants mo Pipol blong Vanuatu. A special thanks for

427 assistanct from Presley Dovo, Frazer Alo, Thomas Doro, Stephanie Sali, Jean-Pascal Wahe, the

428 late Philemon Ala, Marika Tuiwawa, Alivereti Naikatini, Sean Thackurdeen, Jonathan del

429 Rosario, Chanel Sam, Ashley McGuigan, Tamara Ticktin, Tom A. Ranker, Gregory M. Plunkett,

430 and Michael J. Balick. We are extremely grateful to the many communities on Aneityum and

431 Tanna for their kindness, hospitality, and commitment to this project, tankyu tumas. The authors

432 would like to thank the handling editor Jana U'Ren and reviewers Justin Shaffer, Elizabeth

433 Bowman, and anonymous for thoughtful reviews. Nicole Hynson, Tom A. Ranker, Nhu Nguyen,

434 Mikey Kantar, contributed to project design and manuscript improvement. We are also very

435 appreciative of The Vanuatu Department of Forestry for their partnership and logistical support.

436 This study is part of the NSF project, "Collaborative Research: Plant and Fungal Diversity of

437 Tafea Province, Vanuatu, A Threatened Pacific Hotspot. This is publication 106 from the School

438 of Life Sciences, University of Hawai'i at Mānoa

439

440

441

\section{References}

Agrios G. 2004. Plant pathology: Fifth edition. DOI: 10.1016/C2009-0-02037-6.

442 Amend AS, Cobian GM, Laruson AJ, Remple K, Tucker SJ, Poff KE, Antaky C, Boraks A, 
443

444

445

446

447

448

449

450

451

452

453

454

455

456

457

458

459

460

461

462

463

464

465

466

467

468

469

470

471

472

473

474

475

476

477

478

479

480

481

482

483

484

485

486

487

488

Jones CA, Kuehu D, Lensing BR, Pejhanmehr M, Richardson DT, Riley PP. 2019.

Phytobiomes are compositionally nested from the ground up. PeerJ 7:e6609. DOI:

10.7717/peerj.6609.

Bai Y, Müller DB, Srinivas G, Garrido-Oter R, Potthoff E, Rott M, Dombrowski N, Münch PC, Spaepen S, Remus-Emsermann M, Hüttel B, McHardy AC, Vorholt JA, Schulze-Lefert P.

2015. Functional overlap of the Arabidopsis leaf and root microbiota. Nature 528:364-369.

DOI: $10.1038 /$ nature 16192.

Baldrian P. 2017. Forest microbiome: Diversity, complexity and dynamics. FEMS Microbiology Reviews 41:109-130. DOI: 10.1093/femsre/fuw040.

Benjamini Y, Hochberg Y. 1995. Controlling the False Discovery Rate: A Practical and Powerful Approach to Multiple Testing. Journal of the Royal Statistical Society: Series B (Methodological) 57:289-300. DOI: 10.1111/j.2517-6161.1995.tb02031.x.

Bernard J, Wall CB, Costantini MS, Rollins RL, Atkins ML, Cabrera FP, Cetraro ND, Feliciano CKJ, Greene AL, Kitamura PK, Olmedo-Velarde A, Sirimalwatta VNS, Sung HW, Thompson LPM, Vu HT, Wilhite CJ, Amend AS. 2020. Plant part and a steep environmental gradient predict plant microbial composition in a tropical watershed. The ISME Journal. DOI: 10.1101/2020.07.20.212811.

Boraks A, Plunkett GM, Doro TM, Alo F, Sam C, Tuiwawa M, Ticktin T, Amend AS. 2020. Scale-Dependent Influences of Distance and Vegetation on the Composition of Aboveground and Belowground Tropical Fungal Communities. Microbial Ecology. DOI: 10.1007/s00248-020-01608-4.

Brown JH. 1984. On the relationship between abundance and distribution of species. The American Naturalist 124:255-279. DOI: 10.2307/2461494.

Brown JH, Stevens GC, Kaufman DM. 1996. The geographic range: Size, shape, boundaries, and internal structure. Annual Review of Ecology and Systematics 27:597-623. DOI: 10.1146/annurev.ecolsys.27.1.597.

Bruns TD, Peay KG, Boynton PJ, Grubisha LC, Hynson NA, Nguyen NH, Rosenstock NP. 2009. Inoculum potential of Rhizopogon spores increases with time over the first $4 \mathrm{yr}$ of a $99-\mathrm{yr}$ spore burial experiment. New Phytologist 181:463-470. DOI: 10.1111/j.14698137.2008.02652.x.

Carini P, Marsden PJ, Leff JW, Morgan EE, Strickland MS, Fierer N. 2016. Relic DNA is abundant in soil and obscures estimates of soil microbial diversity. Nature Microbiology 2:16242. DOI: 10.1038/nmicrobiol.2016.242.

Chichorro F, Juslén A, Cardoso P. 2019. A review of the relation between species traits and extinction risk. Biological Conservation 237:220-229. DOI: 10.1016/j.biocon.2019.07.001.

Choudoir MJ, Barberán A, Menninger HL, Dunn RR, Fierer N. 2018. Variation in range size and dispersal capabilities of microbial taxa. Ecology 99:322-334. DOI: 10.1002/ecy.2094.

Clark DR, Underwood GJC, Dumbrell AJ, Mcgenity TJ. 2021. What drives study- - dependent differences in distance - - decay relationships of microbial communities ? :1-15. DOI: $10.1111 /$ geb. 13266.

Davis NM, Proctor DM, Holmes SP, Relman DA, Callahan BJ. 2017. Simple statistical identification and removal of contaminant sequences in marker-gene and metagenomics data. bioRxiv:1-14. DOI: 10.1101/221499.

Ferguson BA, Dreisbach TA, Parks CG, Filip GM, Schmitt CL. 2003. Coarse-scale population structure of pathogenic Armillaria species in a mixed-conifer forest in the Blue Mountains of northeast Oregon. Canadian Journal of Forest Research 33:612-623. DOI: 10.1139/x03-

Peer) reviewing PDF | (2021:04:59980:1:1:NEW 9 Jun 2021) 
489

490

491

492

493

494

495

496

497

498

499

500

501

502

503

504

505

506

507

508

509

510

511

512

513

514

515

516

517

518

519

520

521

522

523

524

525

526

527

528

529

530

531

532

533

534

065.

Gaston KJ. 1996. Species-range-size distributions: Patterns, mechanisms and implications. Trends in Ecology and Evolution 11:197-201. DOI: 10.1016/0169-5347(96)10027-6.

Gaston KJ. 2008. Measuring Geographic Range Sizes. Oikos 17:434-438. DOI: 10.1111/j.16000587.1994.tb00094.x.

Gaston KJ, Fuller RA. 2009. The sizes of species' geographic ranges. Journal of Applied Ecology 46:1-9. DOI: 10.1111/j.1365-2664.2008.01596.x.

Gdanetz K, Benucci GMN, Vande Pol N, Bonito G. 2017. CONSTAX: A tool for improved taxonomic resolution of environmental fungal ITS sequences. BMC Bioinformatics 18:1-9. DOI: $10.1186 / \mathrm{s} 12859-017-1952-\mathrm{x}$.

Gloor GB, Macklaim JM, Pawlowsky-Glahn V, Egozcue JJ. 2017. Microbiome Datasets Are Compositional: And This Is Not Optional. Frontiers in Microbiology 8:2224. DOI: 10.3389/fmicb.2017.02224.

Gordon A, Hannon GJ. 2010. Fastx-toolkit. FASTQ/A short-reads preprocessing tools (unpublished) http://hannonlab. cshl. edu/fastx_toolkit 5.

Griffin EA, Carson WP. 2018. Title: Tropical tree endophytes: cryptic drivers of forest diversity, species composition, and ecosystem function.

Halbwachs H, Bässler C. 2015. Gone with the wind - A review on basidiospores of lamellate agarics. Mycosphere 6:78-112. DOI: 10.5943/mycosphere/6/1/10.

Kembel SW, Mueller RC. 2014. Plant traits and taxonomy drive host associations in tropical Phyllosphere fungal communities. Botany 92:303-311. DOI: 10.1139/cjb-2013-0194.

Kivlin SN, Winston GC, Goulden ML, Treseder KK. 2014. Environmental filtering affects soil fungal community composition more than dispersal limitation at regional scales. Fungal Ecology 12:14-25. DOI: 10.1016/j.funeco.2014.04.004.

Love MI, Huber W, Anders S. 2014. Moderated estimation of fold change and dispersion for RNA-seq data with DESeq2. Genome biology 15:550. DOI: 10.1186/s13059-014-0550-8.

Luo Z, Liu J, Zhao P, Jia T, Li C, Chai B. 2019. Biogeographic patterns and assembly mechanisms of bacterial communities differ between habitat generalists and specialists across elevational gradients. Frontiers in Microbiology 10:1-14. DOI: 10.3389/fmicb.2019.00169.

McMurdie PJ, Holmes S. 2013. Phyloseq: An R Package for Reproducible Interactive Analysis and Graphics of Microbiome Census Data. PLOS ONE 8. DOI: 10.1371/journal.pone.0061217.

McMurdie PJ, Holmes S. 2014. Waste Not, Want Not: Why Rarefying Microbiome Data Is Inadmissible. PLoS Computational Biology 10. DOI: 10.1371/journal.pcbi.1003531.

Morton JT, Marotz C, Washburne A, Silverman J, Zaramela LS, Edlund A, Zengler K, Knight R. 2019. Establishing microbial composition measurement standards with reference frames. Nature Communications 10. DOI: 10.1038/s41467-019-10656-5.

Mueller-Dombois D, Fosberg FR. 2013. Vegetation of the tropical Pacific islands. Springer Science \& Business Media.

Nguyen NH, Hynson NA, Bruns TD. 2012. Stayin' alive: Survival of mycorrhizal fungal propagules from 6-yr-old forest soil. Fungal Ecology 5:741-746. DOI: 10.1016/j.funeco.2012.05.006.

O'Rorke R, Cobian GM, Holland BS, Price MR, Costello V, Amend AS. 2015. Dining local: The microbial diet of a snail that grazes microbial communities is geographically structured. Environmental Microbiology 17:1753-1764. DOI: 10.1111/1462-2920.12630. 
535 Oksanen J, Blanchet FG, Kindt R, Legendre P, Minchin PR, O'hara RB, Simpson GL, Solymos

P, Stevens MHH, Wagner H. 2013. Community ecology package. R package version 2
Oono R, Evre EL, Simha A, Lutzoni F, Bonfante P. 2015. A comparison of the community diversity of foliar fungal endophytes between seedling and adult loblolly pines (Pinus taeda). Fungal Biology 119:917-928. DOI: 10.1016/j.funbio.2015.07.003.

Osono T. 2006. Role of phyllosphere fungi of forest trees in the development of decomposer fungal communities and decomposition processes of leaf litter. Canadian Journal of Microbiology 52:701-716. DOI: 10.1139/W06-023.

Peršoh D. 2013. Factors shaping community structure of endophytic fungi-evidence from the Pinus-Viscum-system. Fungal Diversity 60:55-69. DOI: 10.1007/s13225-013-0225-x.

Pickles BJ, Genney DR, Potts JM, Lennon JJ, Anderson IC, Alexander IJ. 2010. Spatial and temporal ecology of Scots pine ectomycorrhizas. New Phytologist 186:755-768. DOI: 10.1111/j.1469-8137.2010.03204.x.

Porras-Alfaro A, Bayman P. 2011. Hidden fungi, emergent properties: endophytes and microbiomes. Annual Review of Phytopathology 49:291-315. DOI: 10.1146/annurev-phyto080508-081831.

Pulliam HR. 2000. On the relationship between niche and distribution. Ecology Letters 3:349361. DOI: 10.1046/j.1461-0248.2000.00143.x.

R Core Team. 2019. R: A language and environment for statistical computing. Accessed 1st April 2019.

Rabinowitz D. 1981. Seven forms of rarity. In: The biological aspects of rare plant conservation. 205-217.

Rivers AR, Weber KC, Gardner TG, Liu S, Armstrong SD. 2018. ITSxpress: Software to rapidly trim internally transcribed spacer sequences with quality scores for marker gene analysis [version 1; peer review: 2 approved]. F1000Research 7:1418. DOI: 10.12688/F1000RESEARCH.15704.1.

Rognes T, Flouri T, Nichols B, Quince C, Mahé F. 2016. VSEARCH: a versatile open source tool for metagenomics. PeerJ 4:e2584. DOI: 10.7717/peerj.2584.

Schoch CL, Seifert KA, Huhndorf S, Robert V, Spouge JL, Levesque CA, Chen W, Bolchacova E, Voigt K, Crous PW, Miller AN, Wingfield MJ, Aime MC, An KD, Bai FY, Barreto RW, Begerow D, Bergeron MJ, Blackwell M, Boekhout T, Bogale M, Boonyuen N, Burgaz AR, Buyck B, Cai L, Cai Q, Cardinali G, Chaverri P, Coppins BJ, Crespo A, Cubas P, Cummings C, Damm U, de Beer ZW, de Hoog GS, Del-Prado R, Dentinger B, DiéguezUribeondo J, Divakar PK, Douglas B, Dueñas M, Duong TA, Eberhardt U, Edwards JE, Elshahed MS, Fliegerova K, Furtado M, García MA, Ge ZW, Griffith GW, Griffiths K, Groenewald JZ, Groenewald M, Grube M, Gryzenhout M, Guo LD, Hagen F, Hambleton S, Hamelin RC, Hansen K, Harrold P, Heller G, Herrera C, Hirayama K, Hirooka Y, Ho HM, Hoffmann K, Hofstetter V, Högnabba F, Hollingsworth PM, Hong SB, Hosaka K, Houbraken J, Hughes K, Huhtinen S, Hyde KD, James T, Johnson EM, Johnson JE, Johnston PR, Jones EBG, Kelly LJ, Kirk PM, Knapp DG, Kõljalg U, Kovács GM, Kurtzman CP, Landvik S, Leavitt SD, Liggenstoffer AS, Liimatainen K, Lombard L, Luangsa-ard JJ, Lumbsch HT, Maganti H, Maharachchikumbura SSN, Martin MP, May TW, McTaggart AR, Methven AS, Meyer W, Moncalvo JM, Mongkolsamrit S, Nagy LG, Nilsson RH, Niskanen T, Nyilasi I, Okada G, Okane I, Olariaga I, Otte J, Papp T, Park D, Petkovits T, Pino-Bodas R, Quaedvlieg W, Raja HA, Redecker D, Rintoul TL, Ruibal C, Sarmiento-Ramírez JM, Schmitt I, Schüßler A, Shearer C, Sotome K, Stefani FOP, Stenroos 
581

582

583

584

585

586

587

588

589

590

591

592

593

594

595

596

597

598

599

600

601

602

603

604

605

606

607

608

609

610

611

612

613

614

615

616

617

618

619

620

621

622

623

624

625

626

S, Stielow B, Stockinger H, Suetrong S, Suh SO, Sung GH, Suzuki M, Tanaka K, Tedersoo L, Telleria MT, Tretter E, Untereiner WA, Urbina H, Vágvölgyi C, Vialle A, Vu TD, Walther G, Wang QM, Wang Y, Weir BS, Weiß M, White MM, Xu J, Yahr R, Yang ZL, Yurkov A, Zamora JC, Zhang N, Zhuang WY, Schindel D. 2012. Nuclear ribosomal internal transcribed spacer (ITS) region as a universal DNA barcode marker for Fungi. Proceedings of the National Academy of Sciences of the United States of America. DOI: 10.1073/pnas.1117018109.

Sheth SN, Morueta-Holme N, Angert AL. 2020. Determinants of geographic range size in plants. New Phytologist 226:650-665. DOI: 10.1111/nph.16406.

Slatyer RA, Hirst M, Sexton JP. 2013. Niche breadth predicts geographical range size: A general ecological pattern. Ecology Letters 16:1104-1114. DOI: 10.1111/ele.12140.

Suryanarayanan TS, Thennarasan S. 2004. Temporal variation in endophyte assemblages of Plumeria rubra leaves. :197-204.

Unterseher M, Gazis R, Chaverri P, Guarniz CFG, Tenorio DHZ. 2013. Endophytic fungi from Peruvian highland and lowland habitats form distinctive and host plant-specific assemblages. Biodiversity and Conservation 22:999-1016. DOI: 10.1007/s10531-013-0464$\mathrm{x}$.

Unterseher M, Karunarathna SC, Cruz GR, Dagamac NH, Dahl MB, Dool SE, Galla M, Herbst L, Nilsson RH, Puechmaille SJ, Schöner C, Schöner M, Siddique AB, Teltewskoi A, Wicke K, Würth DG, Wurzbacher C, Hyde KD. 2018. Mycobiomes of sympatric Amorphophallus albispathus (Araceae) and Camellia sinensis (Theaceae) - a case study reveals clear tissue preferences and differences in diversity and composition. Mycological Progress 17:489500. DOI: 10.1007/s11557-018-1375-8.

Vanuatu Meteorology and Geo-hazard Department, Australian Bureau of Meteorology, CSIRO. 2015. Current and future climate of Vanuatu. Melbourne, Australia.

Větrovský T, Morais D, Kohout P, Lepinay C, Algora C, Awokunle Hollá S, Bahnmann BD, Bílohnědá K, Brabcová V, D’Alò F, Human ZR, Jomura M, Kolařík M, Kvasničková J, Lladó S, López-Mondéjar R, Martinović T, Mašínová T, Meszárošová L, Michalčíková L, Michalová T, Mundra S, Navrátilová D, Odriozola I, Piché-Choquette S, Štursová M, Švec K, Tláskal V, Urbanová M, Vlk L, Voř́̌šková J, Žifčáková L, Baldrian P. 2020. GlobalFungi, a global database of fungal occurrences from high-throughput-sequencing metabarcoding studies. Scientific Data 7:1-14. DOI: 10.1038/s41597-020-0567-7.

Vorholt JA. 2012. Microbial life in the phyllosphere. Nature reviews. Microbiology 10:828-40. DOI: $10.1038 /$ nrmicro2910.

Wagner MR, Lundberg DS, del Rio TG, Tringe SG, Dangl JL, Mitchell-Olds T. 2016. Host genotype and age shape the leaf and root microbiomes of a wild perennial plant. Nat Commun 7:1-15. DOI: 10.1038/ncomms12151.

Wildman HG, Parkinson D. 1979. Microfungal succession on living leaves of Populus tremuloides. Canadian Journal of Botany 57:2800-2811. DOI: 10.1139/b79-332.

Zarraonaindia I, Owens SM, Weisenhorn P, West K, Hampton-Marcell J, Lax S, Bokulich NA, Mills DA, Martin G, Taghavi S, van der Lelie D, Gilbert JA. 2015. The soil microbiome influences grapevine-associated microbiota. mBio 6:1-10. DOI: 10.1128/mBio.02527-14.

Žifčáková L, Větrovský T, Howe A, Baldrian P. 2016. Microbial activity in forest soil reflects the changes in ecosystem properties between summer and winter. Environmental Microbiology 18:288-301. DOI: 10.1111/1462-2920.13026.

Peer] reviewing PDF | (2021:04:59980:1:1:NEW 9 Jun 2021) 


\section{Table $\mathbf{1}$ (on next page)}

Inter-habitat dissimilarity measurements comparing paired soil-phyllosphere communities ${ }^{\triangle}$ and a null hypothesis ${ }^{\Omega}$.

Spatial autocorrelation is indicated by the smaller dissimilarities between observed communities than the null-hypothesis. Significance was tested by student t-test. The entire study spanned $<110 \mathrm{~km}$, and the six transects (T1 - T12) each covered an area of $400 \mathrm{~m}^{2}$. 
1

\begin{tabular}{|c|c|c|c|c|c|c|c|}
\hline & \multicolumn{7}{|c|}{ Transect } \\
\hline & Entire study & T1 & T4 & T6 & T9 & T10 & T12 \\
\hline Distance & $(<110 \mathrm{~km})$ & \multicolumn{7}{|c|}{ Bray-Curtis dissimilarity (Soil-Phyllosphere) } \\
\hline & \multicolumn{8}{|c|}{ (10 $)$} \\
\hline Observed $^{\Delta}$ & $\mathbf{0 . 9 2 5}^{* *}$ & 0.926 & 0.881 & $\mathbf{0 . 9 3 4}^{*}$ & $\mathbf{0 . 9 2 9}^{*}$ & $\mathbf{0 . 9 2 8}^{* * *}$ & 0.960 \\
\hline Randomized $^{\Omega}$ & $\mathbf{0 . 9 5 8}^{\text {***}}$ & 0.931 & 0.893 & $\mathbf{0 . 9 4 8}^{*}$ & $\mathbf{0 . 9 4 0}^{*}$ & $\mathbf{0 . 9 4 3}^{* * *}$ & 0.964 \\
\hline
\end{tabular}

$2 \Delta$ mean BC dissimilarity for fungal communities from soil and phyllosphere of the identical sampling site

$3 \Omega$ mean $B C$ dissimilarity for fungal communities from soil and phyllosphere of a randomized sampling site

4 p-value: $<0.001^{* * * *} ;<0.01^{* * * *} ;<0.05^{*}$

5

6 


\section{Figure 1}

A comparison of soil and phyllosphere fungal communities.

A comparison of soil and phyllosphere fungal communities. NMDS ordination (a) indicating differences between fungal communities based on Bray-Curtis dissimilarity. Each point is a fungal community sequenced from the soil (circle) and phylloplane (triangle). Ellipses represent 95\% confidence intervals around the centroids. Separation along axis 2 corresponded to difference in islands and sampling date. Permanova : habitat $\left(R^{2}=0.11, p\right.$ $<0.001)$ and island/date $\left(R^{2}=0.03, p<0.001\right)$. Venn diagram (b) emphasizing the distribution of OTU generalist and specialists for each habitat (observed richness). Singleton OTU numbers are excluded from diagram.
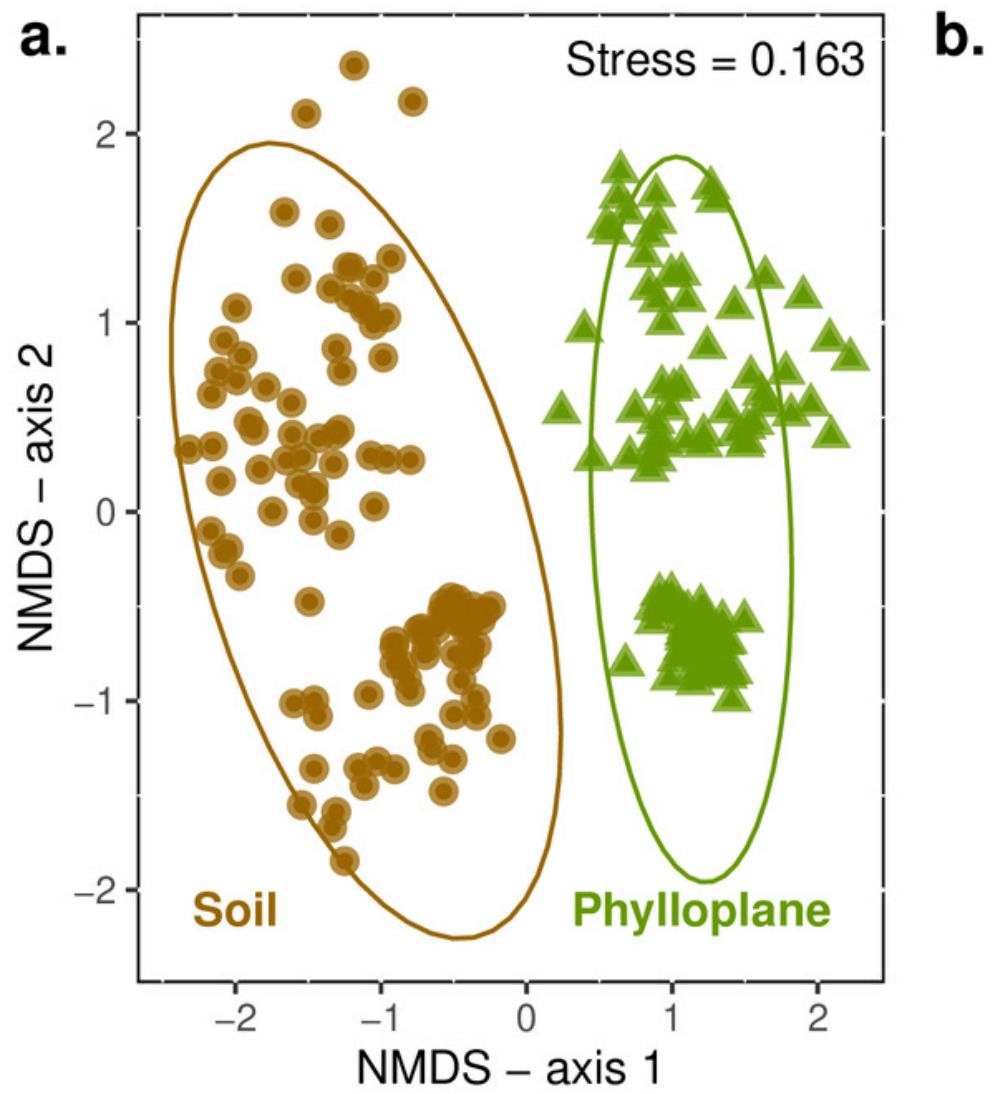

Phylloplane specialists

2212

Generalists

1307

Soil specialists

2187 


\section{Figure 2}

Transect occupancy measured by the number of times an OTU occurred within a transect.

Transect occupancy measured by the number of times an OTU occurred within a transect. Histogram bars represent a tally of the OTU density for particular transect occupancy levels (y-axis). Occupancy (x-axis) is measured as the number of occurrences per transects (max 36). Generalist fungi occur more commonly than specialist fungi as is indicated by the proportion of generalists found on the right-hand side of the histogram. The number of occurrences for each habitat and specialization pair differed significantly ( $p<0.001$ ) as determined by pairwise Wilcoxon test corrected for multiple hypothesis testing. Y-axis is on a $\log _{10}$ scale.

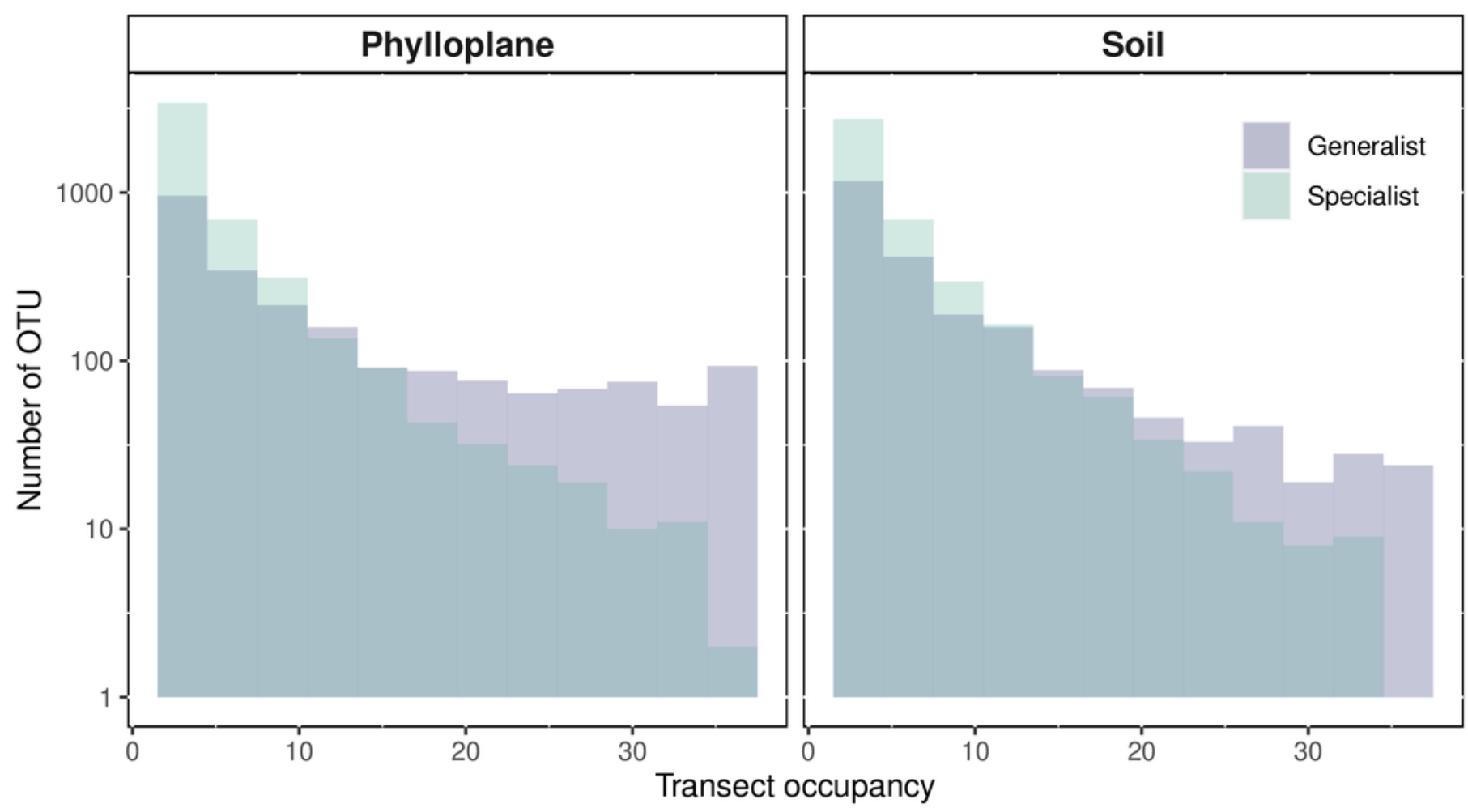




\section{Figure 3}

Range size (meters) for individual fungi of the soil and phyllosphere as calculated from the range of semi-variogram models.

Specialist fungi occurred more than once and exclusively within a single habitat, whereas Generalist fungi were found in both habitats. While the identities of soil and phyllosphere generalist OTUs are the same, the range size estimated for each OTU differs per habitat. Each dot represents the range size for a single fungal OTU. The colored areas indicate the probability distribution of range sizes. OTU abundance is measured as a function of relative sequence read abundance. Outliers with large range sizes are not presented here but can be found in Supplemental Table S1.
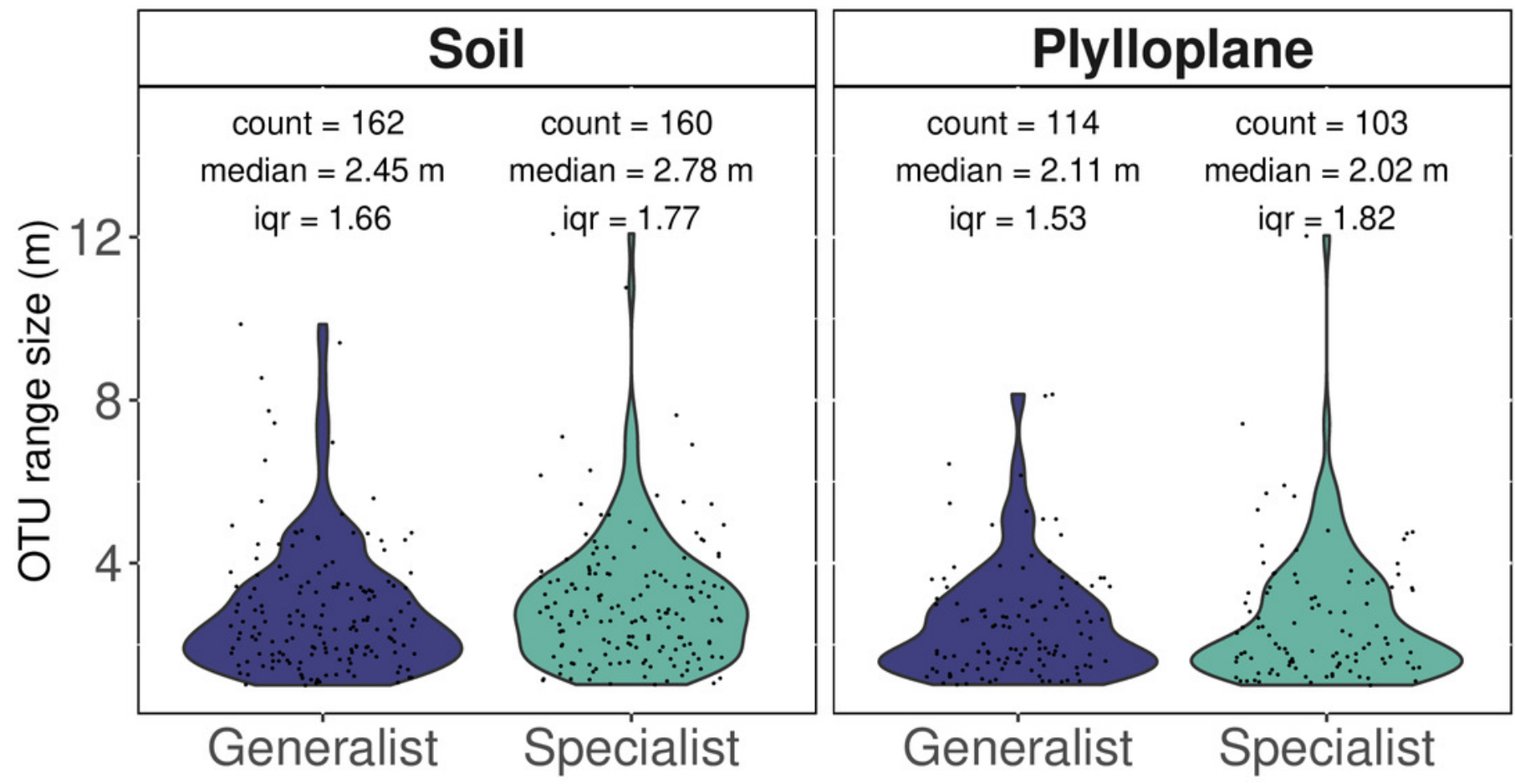


\section{Figure 4}

Generalist fungi that occur in soil are often found nearby in the phyllosphere.

Maps detailing the distribution of 11 fungal OTUs. Each set of maps illustrates the distribution of OTU sequence read abundance within a transect ( $10 \mathrm{~m}$ by $40 \mathrm{~m}$ ). Maps are coupled so that the distribution of an OTU from soil is paired with the same OTU from the phyllosphere of the same transect. Dark colors indicate low abundance and light colors indicate high abundance. The OTUs were identified as: a. Dothideomycetes sp.; b. Helotiales sp,; c. Agaricomycetes sp.; d. Psathyrella sp.; e. Polyporale sp.; f. Xylariales sp.; g. Agaricales sp.; h. Psathyrellaceae sp.; i. Psathyrellaceae sp.; j. Xylariales sp.; k. Ascomycota sp.

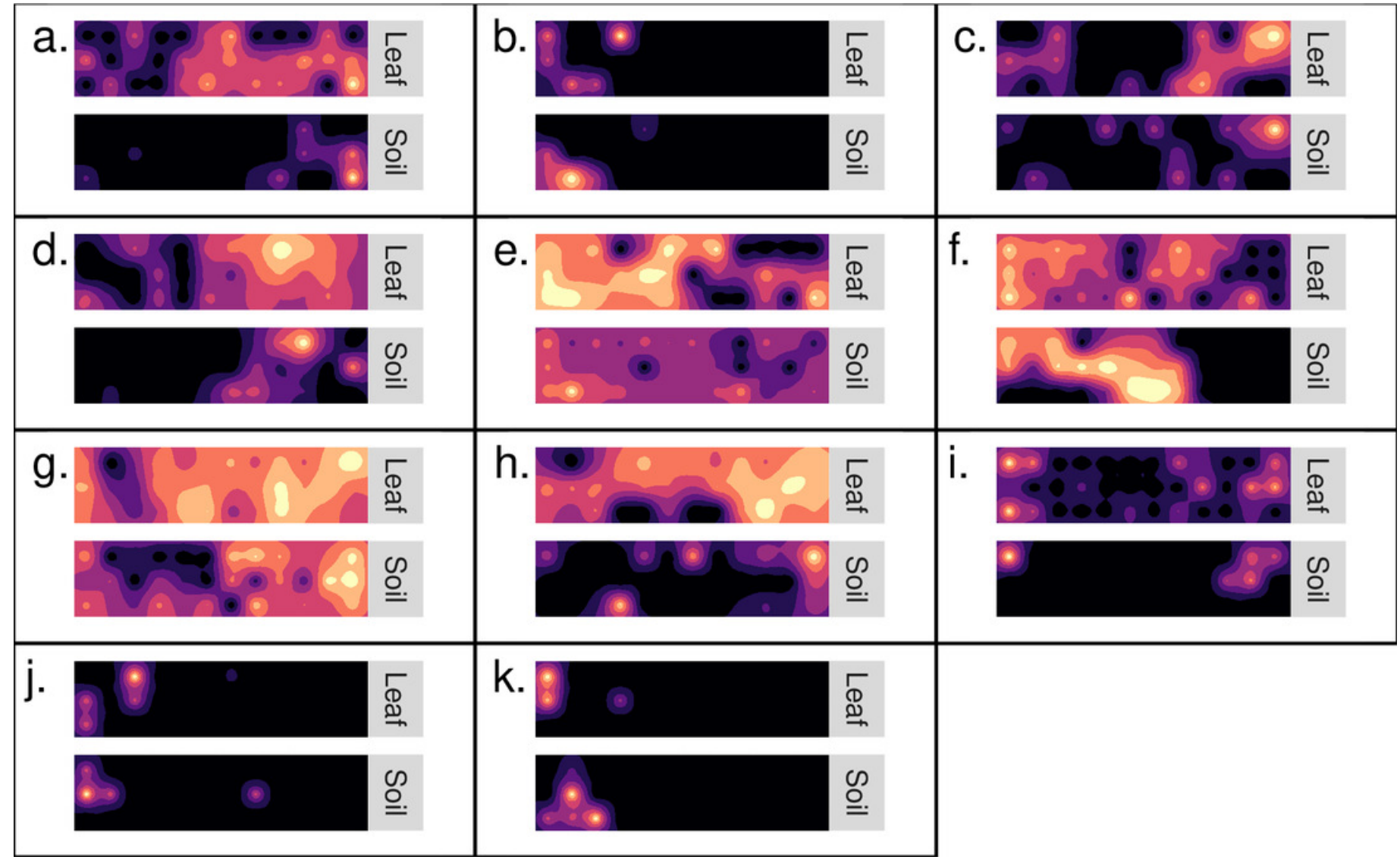




\section{Figure 5}

Interspecific occupancy-abundance relationships for OTUs of the soil and phyllosphere.

Interspecific occupancy-abundance relationships for OTUS. Soil specialists are more abundant than soil generalists at low occupancy levels. Phyllosphere specialists and generalists diverge at high occupancy levels. Occupancy ( $x$-axis) is the number of sampling sites, within a single transect, that an OTU was found. Local abundance (y-axis) is the sum of an OTU's sequence read abundance (VST corrected) divided by the occupancy rate.

Regression fitted with a loess curve and shaded ribbons show the $95 \%$ confidence interval

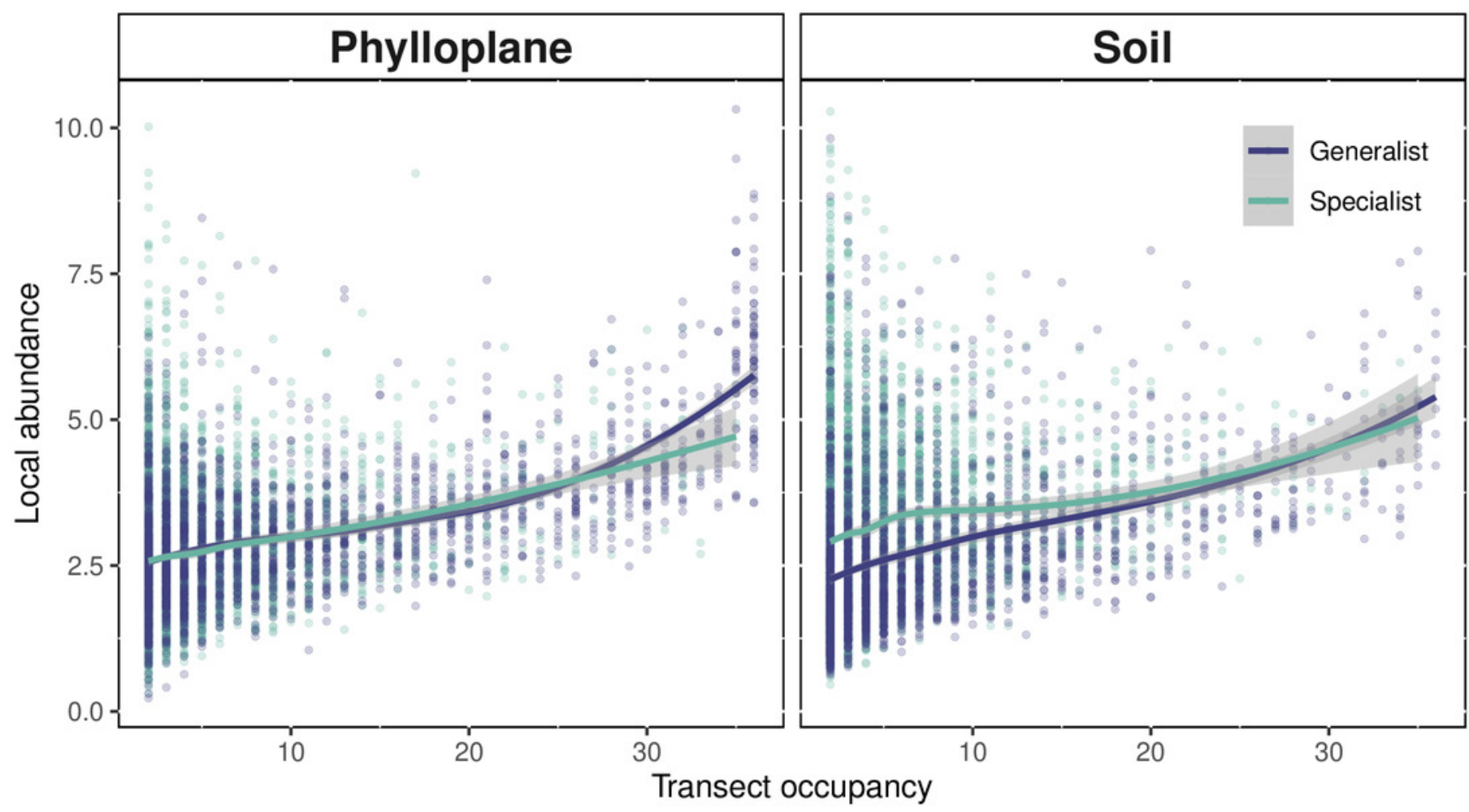

